\title{
miR-199a-3p Inhibits Aurora Kinase A and Attenuates Prostate Cancer Growth
}

\section{New Avenue for Prostate Cancer Treatment}

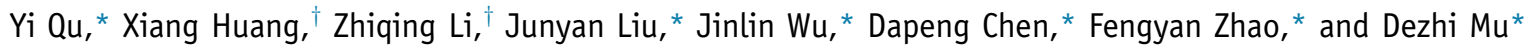

From the Key Laboratory of Birth Defects of Ministry of Education, ${ }^{*}$ Sichuan University, Chengdu; and the Department of Urology, ${ }^{\dagger}$ The People's Hospital of Sichuan Province, Chengdu, China

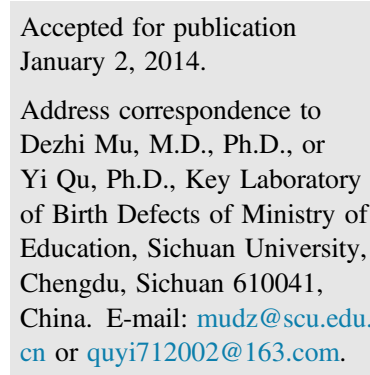

Prostate cancer ( $\mathrm{PCa}$ ) is the most common solid tumor malignancy in men that severely influences quality of life. Surgery is most often the recommended treatment for $\mathrm{PCa}$, but radical prostatectomy can cause significant urinary adverse effects. Therefore, finding effective biochemical treatments for PCa remains a necessity. Aurora kinase A has been shown to be involved in PCa progression, thus making it a good target for PCa therapy. miRNAs are important regulators of gene expression, with some miRNAs specifically involved in carcinogenesis. Therefore, herein, we identified miRNAs targeted to aurora kinase $A$ and examined their effects on the growth of PCa. We used primary samples from PCa patients and PCa cell lines as research subjects. We demonstrate that miR-199a-3p is down-regulated in PCa tissues compared with normal prostate tissues, with the expression pattern inversely correlated with the expression pattern of aurora kinase A. We find that miR-199a-3p agomir inhibits aurora kinase A and attenuates xenograft tumor growth of PCa. Moreover, we demonstrate that down-regulation of miR-199a-3p results from enhancement of the methylation of miR-199a gene in PCa. Furthermore, we find that the expression level of miR-199a-3p is inversely correlated to tumor stage and Gleason score of PCa. Revealing novel mechanisms for oncogene inhibition by miRNA-mediated pathways offers new avenues for PCa treatment. (Am J Pathol 2014, 184: 1541-1549; http://dx.doi.org/10.1016/j.ajpath.2014.01.017)
Prostate cancer (PCa) is the most common solid tumor malignancy in men that severely influences quality of life. However, the treatment for PCa remains challenging. Surgery is most often the recommended treatment for young men, but radical prostatectomy can cause significant urinary adverse effects. Therefore, finding effective biochemical treatments for PCa remains a necessity.

Recently, aurora kinase A has been demonstrated to be involved in PCa progression and enhance cancer growth and migration. ${ }^{1,2}$ Furthermore, our previous work demonstrated that aurora kinase A up-regulation was closely associated with malignant phenotypes in $\mathrm{PCa}$, suggesting that aurora kinase A may be a good target for PCa therapy. ${ }^{3}$

To inhibit aurora kinase A and the subsequently induced malignant phenotypes, we need to clarify the mechanism by which aurora kinase $\mathrm{A}$ is up-regulated in $\mathrm{PCa}$. In recent years, a new class of molecules has been found to regulate gene expression, known as miRNA. ${ }^{4}$ miRNAs are a family of small (approximately 22 nucleotides), endogenous, noncoding RNAs that, by binding complementary sequences in the $3^{\prime}$ untranslated region $\left(3^{\prime}\right.$-UTR) of mRNAs, either mediate translational repression or direct mRNA cleavage. Thus, the translation of these mRNAs is inhibited, or they are destabilized, resulting in down-regulation of the encoded protein. ${ }^{5}$ A wide variety of miRNAs have been proved to

\footnotetext{
Supported by National Science Foundation of China grants 81330016 and 31171020 (D.M.) and 81172174 and 81270724 (Y.Q.), the Major State Basic Research Development Program grant 2013CB967404, Ministry of Education of China grants 313037 and 20110181130002 , State Commission of Science Technology of China grant 2012BAI04B04, Science and Technology Bureau of Sichuan province grants 2010SZ0280 and 2011JTD0005, and Program for Outstanding Young Scholars of Sichuan University grant 2012SCU04B03.
}

Disclosures: None declared. 
contribute to malignant phenotypes in cancer. ${ }^{6}$ Therefore, we hypothesize that regulation of some specific miRNAs could play a crucial role in the up-regulation of aurora kinase A in PCa. In the present study, we try to select and identify miRNAs targeted to aurora kinase $\mathrm{A}$, and prove that these miRNAs regulate aurora kinase A in PCa. Meanwhile, because miRNAs are highly stable and compatible with tissues, ${ }^{7}$ we explore the feasibility of using miRNAs to inhibit PCa growth both in vitro and in vivo.

\section{Materials and Methods}

\section{Human PCa Specimens}

Surgical specimens used in this study were collected at The People's Hospital of Sichuan Province (Sichuan, China) from 2007 to 2011. Procedures were in accordance with the Declaration of Helsinki of 1975 and approved by the Ethics Committee of Human Experimentation of Sichuan University (Sichuan). Specimens were selected from the prostate tissue bank. Blocks were manually trimmed to further enrich the histological features of interest. Sections $(8 \mu \mathrm{m}$ thick) were prepared from each block. The first and last sections for each block were subjected to H\&E staining and used to calculate epithelium percentage. The tumor content in the tumor specimens was $>80 \%$, whereas normal samples had at least $60 \%$ epithelium content and no evidence of tumor present. We acquired 12 cases of paired benign and tumor prostate tissues from patients with a Gleason score of $\geq 7$. In these cases, matched normal and tumor samples from the same patients were available. We also acquired unpaired 50 $\mathrm{PCa}$ tissues and 20 normal prostate tissues.

\section{Cell Culture and Transfection}

Human PCa cell lines PC-3, DU145, and LNCaP were purchased from ATCC (Manassas, VA), and cultured in RPMI 1640 medium, supplemented with $10 \%$ fetal bovine serum, $1 \% \mathrm{~L}$-glutamine, and $1 \%$ penicillin-streptomycin. All cell cultures were incubated at $37^{\circ} \mathrm{C}$ in $5 \% \mathrm{CO}_{2}$ and $95 \%$ air. For cell transfection, DU145 cells were seeded at a density of $1.0 \times 10^{5}$ (24-well plates). The dosage of miRNA agomir or antagomir (RiboBio, Guangzhou, China) used was determined according to the manufacturer's protocol. miR-199a-3p agomir (4 nmol/L, final concentration) or miR-199a-3p antagomir (20 nmol/L, final concentration) in $50 \mu \mathrm{L}$ of Opti-MEM was complexed with $1 \mu \mathrm{L}$ of transfection reagent (Lipofectamine) in $50 \mu \mathrm{L}$ of Opti-MEM (Ambion, Austin, TX). The cells were transfected with these complexes and maintained for 8 hours, then fed with original medium. In all experiments, an equal concentration of nontargeting controls (agomir- or antagomir-negative controls) was used as controls for non-sequence-specific effects, whereas Lipofectamine was used as the vehicle control. At 24 and 48 hours after transfection, the cells were collected for detection.

\section{RT-PCR Data}

Total RNA was extracted from both PCa cells cultured in vitro and prostate tissues using an miRNeasy Mini Kit (Qiagen, Hilden, Germany), and from formalin-fixed, paraffinembedded (FFPE) tissues using an miRNeasy FFPE Kit (Qiagen), according to the manufacturer's protocol. The cDNA was synthesized from $2 \mu \mathrm{g}$ of total RNA with Moloney Murine Leukemia Virus (M-MLV) Reverse Transcriptase (Promega, Madison, WI) in a $25-\mu \mathrm{L}$ volume $[2 \mu \mathrm{g}$ total RNA, $400 \mathrm{mmol} / \mathrm{L}$ reverse transcription primer (random primers for U6 rRNAand miRNA-specific primers for miRNA), $4 \mathrm{U} / \mu \mathrm{L}$ M-MLV, and $0.4 \mathrm{mmol} / \mathrm{L}$ dNTP mix]. Real-time PCR was performed with SYBR Green I mix (Takara, Tokyo, Japan) in a $20-\mu \mathrm{L}$ reaction volume $(10 \mu \mathrm{L}$ SYBR Green I mix, $200 \mathrm{mmol} / \mathrm{L}$ forward and reverse primer, and $2 \mu \mathrm{L}$ cDNA template) on an MJ Opticon Monitor chromo4 instrument (Bio-Rad, Hercules, CA) using the following protocol: $95^{\circ} \mathrm{C}$ for 20 seconds and 40 cycles of $95^{\circ} \mathrm{C}$ for 10 seconds, $60^{\circ} \mathrm{C}$ for 20 seconds, and $70^{\circ} \mathrm{C}$ for 1 second. Data analysis was performed using the $2^{-\Delta \Delta C_{T}}$ method. ${ }^{8}$ The primers for miRNA were designed according to an miRNA primer designer (http://www.leonxie.com/ miRNAprimerDesigner.php, last accessed March 11, 2014).

The primers for hsa-miR-199a-3p were 5'-GTCGTATCCAGTGCAGGGTCCGAGGTATTCGCACTGGATACGACTAACCA-3' (reverse transcription), 5'-GCGGCGGACAGTAGTCTGCAC-3' (forward), and 5'-ATCCAGTGCAGGGTCCGAGG-3' (reverse).

The primers for U6 rRNA were purchased from RiboBio.

\section{Western Blot Analysis}

Proteins of cultured cells or prostate tissues were prepared by suspending cell pellets or grinding tissues in lysis buffer (50 $\mathrm{mmol} / \mathrm{L}$ Tris- $\mathrm{HCl}, 150 \mathrm{mmol} / \mathrm{L} \mathrm{NaCl}, 1 \% \mathrm{NP} 40,0.5 \%$ deoxycholate, and $0.1 \%$ SDS), including proteinase inhibitors ( $1 \mathrm{mg} / \mathrm{mL}$ aprotinin, $1 \mathrm{mg} / \mathrm{mL}$ leupeptin, and $1 \mathrm{mmol} / \mathrm{L}$ phenylmethylsulphonyl fluoride). Proteins of FFPE tissues were extracted using a Qproteome FFPE Tissue Kit (Qiagen), according to the manufacturer's protocol. Protein concentration was measured using a DcProtein Assay Kit (Bio-Rad). Equal amounts of protein $(40 \mathrm{mg})$ were loaded onto an SDSpolyacrylamide gel for electrophoresis and then transferred to a polyvinylidene fluoride membrane (Amersham, Uppsala, Sweden). The membrane was then incubated with primary antibody for 1 hour at room temperature. After washing with Tris-buffered saline with Tween 20 , the membrane was incubated with corresponding horseradish peroxide-conjugated secondary antibody (Amersham) and the signals were visualized by electrochemiluminescence (Amersham). The primary antibodies used included anti-aurora $\mathrm{A}$ antibody to detect aurora kinase A (1:800; Santa Cruz Biotechnology, Santa Cruz, CA) and anti-active caspase-3 to detect phosphorylated caspase-3 (1:1000; Chemicon, Bedford, MA). An NIH image was used to measure the densities of the protein signals, and the protein levels were normalized to $\beta$-actin as an internal control. 


\section{Luciferase Reporter Assay}

The miR-199a-3p binding site of aurora kinase A was amplified by PCR from genomic DNA of the human DU145 cell line, which was extracted with a QIAamp DNA Mini Kit (Qiagen). The PCR products were gel purified, digested, and inserted into the pMIR-RB-REPORT vector (Ribobio), and the resulting plasmid was labeled pWT. The miR-199a-3p binding site mutations (pMUT) were introduced using Multisite-Quickchange (Stratagene, San Diego, CA), according to the manufacturer's protocol, and cloned into the pMIRRB-REPORT vector (Ribobio). All inserted or mutated sequences were confirmed by sequencing. HeLa cells were transfected with miRNA-mimics and pWT using Lipofectamine. Meanwhile, miRNA-mimics and pMUT, miRNAcontrol, and pWT, or miRNA-control and pMUT were also transfected in Hela cells. The cells were lysed 48 hours after the transfection for measurement of luciferase activity. A dualluciferase assay was used to quantitate the effects of miR-199a$3 p$ interaction with the $3^{\prime}$-UTR of aurora kinase A. In all experiments, transfection efficiencies were normalized to hLuc luciferase, which was constitutively expressed by the pMIRRB-REPORT vector.

\section{Cell Cycle Distribution Detected by Flow Cytometry}

To detect cell cycle distribution, cells were collected and washed with PBS, and then suspended in Krishan buffer ( $0.1 \%$ sodium citrate, $0.2 \mathrm{mg} / \mathrm{mL}$ RNAase, and $0.3 \% \mathrm{NP} 40$ ) containing $0.05 \mathrm{mg} / \mathrm{mL}$ propidium iodide and then incubated at $4^{\circ} \mathrm{C}$ for 30 minutes. Cells were subjected to Coulter flow cytometry for analysis. Data were analyzed using ModFitLT 2.0 (Verity Software House, Topsham, ME).

\section{Tumor Xenograft Mouse Models}

These studies were approved by the Animal Care and Ethics Committee of Sichuan University. In total, 30 five-week-old male BALB/C nude mice with an average weight of $20 \mathrm{~g}$ were housed in separate cages. DU145 cells were s.c. transplanted into each mouse. After 5 weeks, the tumor tissues had increased to a volume of approximately $65 \mathrm{~mm}^{3}$ and were ready to use for the following experiments. Nude mice were assigned to three groups, each consisting of 10 mice. The first group, serving as a control, received an equal volume $(30 \mu \mathrm{L})$ of PBS injection, but no miRNA treatment. Each mouse in the second group was injected with $10 \mathrm{nmol} / \mathrm{L}$ of agomirs (dissolved in $30 \mu \mathrm{L}$ PBS) into the tumor tissue every 2 days by multipoint intratumoral injection. Each mouse in the third group received $10 \mathrm{nmol} / \mathrm{L}$ of negative agomir control (dissolved in $30 \mu \mathrm{L} \mathrm{PBS}$ ) in the same way as the other two groups. Tumor volumes were assessed using digital Vernier calipers every 2 days in a blinded way (Y.Q., D.M.), and each tumor volume (in $\mathrm{mm}^{3}$ ) was calculated by the following formula:

$$
\mathrm{V}=0.4 \times \mathrm{D} \times \mathrm{d}^{2}
$$

where $\mathrm{V}$ is volume, $\mathrm{D}$ is longitudinal diameter, and $\mathrm{d}$ is latitudinal diameter.

Fifteen days later, mice in each group were sacrificed. The body weight of the mice and the wet weight of the tumors were measured. The tumor tissues were then collected to determine the expressions of aurora kinase $\mathrm{A}$ and active caspase-3 using Western blot analysis.

\section{AZA Treatment}

DU145 cells were seeded at a density of $5 \times 10^{6} / \mathrm{mL}$, and the DNA methylation inhibitor, 5-azacytidine (AZA; Merck, Bedford, MA), was added at a concentration of $1 \mu \mathrm{mol} / \mathrm{L}$ and incubated for 72 hours. Fresh medium was added every 24 hours, with or without AZA.

\section{Genomic DNA Extraction and Bisulfite Modification}

Genomic DNA from culture cells was extracted with the QIAamp DNA mini kit (Qiagen) and from FFPE tissues with the QIAamp DNA FFPE Tissue Kit (Qiagen). The DNA (4 $\mu \mathrm{g}$ in a volume of $100 \mu \mathrm{L}$ ) was sheared 60 times with a $1-\mathrm{mL}$ syringe, then $1 \mu \mathrm{g}$ in a $50-\mu \mathrm{L}$ volume was denatured by $\mathrm{NaOH}$ (final concentration, $0.27 \mathrm{~mol} / \mathrm{L}$ ) for 25 minutes at $37^{\circ} \mathrm{C}$. Then, $30 \mu \mathrm{L}$ of freshly prepared $10 \mathrm{mmol} / \mathrm{L}$ hydroquinone and 520 $\mu \mathrm{L}$ of $3 \mathrm{~mol} / \mathrm{L}$ sodium bisulfite (containing $0.21 \mathrm{~mol} / \mathrm{L}$ $\mathrm{NaOH}$ ) were added, and the mixture was incubated under mineral oil at $50^{\circ} \mathrm{C}$ for 16 hours. Modified DNA was purified using the Axygen gel recovery kit, according to the manufacturer's instructions, and eluted into $50 \mu \mathrm{L}$. Modification was completed by $\mathrm{NaOH}$ (final concentration, $0.27 \mathrm{~mol} / \mathrm{L}$ ) treatment for 20 minutes at $37^{\circ} \mathrm{C}$, followed by ethanol precipitation. The bisulfite-treated DNA was resuspended in 20 $\mu \mathrm{L}$ of water and used immediately or stored at $-20^{\circ} \mathrm{C}$.

\section{MSP Data}

According to Cheung et al, ${ }^{9}$ a hypermethylated region was mapped to the $m i R-199 a$ gene and its upstream promoter (234 to 471), which leads to the down-regulation of miR-199a in testicular tumors. Therefore, we searched for $\mathrm{CpG}$ sites $400 \mathrm{bp}$ upstream and $200 \mathrm{bp}$ downstream from the $m i R-199 a$ gene, and designed primers for methylation-specific PCR (MSP) using Methprimer (http://www.urogene.org/methprimer, last accessed March 11, 2014) (Table 1). The methylation status of this region was determined using an SYBR Green-based quantitative methylation-specific PCR (qMSP).

In brief, genomic DNA was extracted from cells or tissues, and modified by bisulfite, as previously described. For PCR (final volume, $20 \mu \mathrm{L}$ ), $0.5 \mu \mathrm{L}$ of bisulfite-treated DNA template was mixed with $10 \mu \mathrm{L}$ of $2 \times$ Power SYBR Green PCR Master Mix (Applied Biosystems, Foster, CA) plus primers to a final concentration of $200 \mathrm{nmol} / \mathrm{L}$. The PCR conditions included an initial incubation at $50^{\circ} \mathrm{C}$ for 2 minutes, denaturing at $95^{\circ} \mathrm{C}$ for 10 minutes, and 40 cycles of denaturing at $95^{\circ} \mathrm{C}$ for 15 seconds and annealing at $60^{\circ} \mathrm{C}$ for 1 minute. After 
Table 1 Primers for MSP of hsa-miR-199a

\begin{tabular}{lll}
\hline Primer name & Primer sequences & Product size (bp) \\
\hline Left M primer & $5^{\prime}$-AGGGAGGTTTTTTTTGAGGATC-3' & 134 \\
Right M primer & $5^{\prime}$-CCAATATACAAACTACTATACAACGAC-3' \\
Left U primer & $5^{\prime}$-GGAGGTTTTTTTTGAGGATTG-3' & 132 \\
Right U primer & $5^{\prime}$-CCAATATACAAACTACTATACAACAAC-3' & \\
\hline
\end{tabular}

M, methylated; $U$, unmethylated.

PCR amplification, a dissociation curve was generated to confirm the size of the PCR product. MSP reactions were optimized using methylated (methylated positive control) and unmethylated (unmethylated positive control) bisulfite-converted control DNA (Epitect control DNA set; Qiagen). Amplification products were quantified by counting $\mathrm{C}_{\mathrm{T}}$ values and visualized by UV illumination on a $2 \%$ agarose gel.

The methylation percentage was estimated using the following formula:

$$
\begin{aligned}
& \text { methylated }(\%)=1 / 1+2^{(-\Delta \mathrm{Ct})} \\
& \Delta \mathrm{Ct}=\mathrm{CtU}-\mathrm{CtM},
\end{aligned}
$$

where $M$ is the copy number of methylated miR-199a, and $\mathrm{U}$ is the copy number of unmethylated miR-199a.

\section{Statistics}

Data are presented as means \pm SEM from three independent experiments. A Student's $t$-test was used when comparing between two groups. An analysis of variance with Fisher's post hoc test was used when comparing more than two groups. The correlation coefficient was analyzed by Spearman's rank correlation. $P<0.05$ was defined as the threshold for significance.

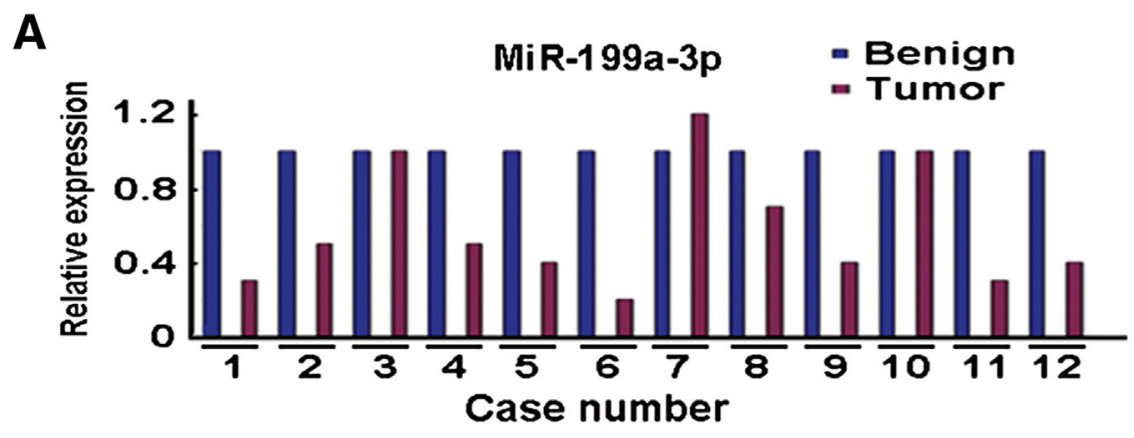

B

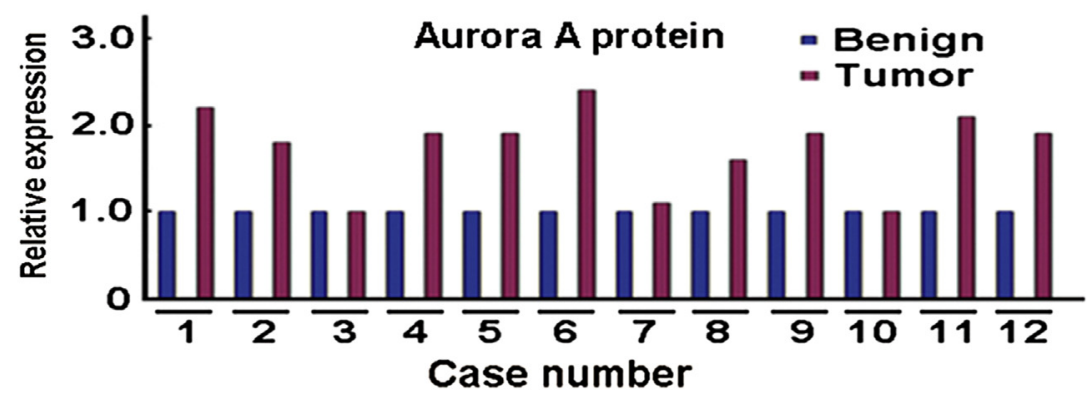

C

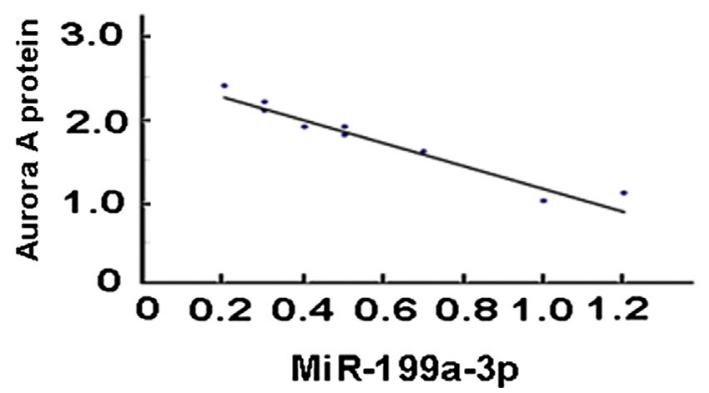

Figure 1 miR-199a-3p expression is downregulated in human PCa. A: Relative expression of miR-199a-3p in the 12 paired benign and tumor human prostate samples. The data of miR-199a-3p expression in benign or tumor prostate tissues from three independent sets of RT-qPCR were averaged, the data for benign tissues were arbitrarily designated as one, and the relative expression was obtained when data for tumor tissues were divided by data for benign tissues. B: Relative expression of aurora kinase A protein in the 12 paired benign and tumor human prostate samples. The data of aurora kinase A protein in benign or tumor prostate tissues from three independent sets of Western blot analysis experiments were averaged, the data for benign tissues were arbitrarily designated as one, and the relative expression was obtained when data for tumor tissues were divided by data for benign tissues. C: Correlogram using Excel shows that expression of aurora kinase $A$ protein correlates inversely with that of miR-199a-3p $\left(R^{2}=0.938\right)$. 


\section{Results}

\section{Identification of miRNAs Binding to Aurora Kinase A mRNA}

To assess the miRNAs that can target aurora kinase A, a bioinformatic search was performed in the miRandal miRDB\miRWalk\RNA22ltargetscan databases (http://www. targetscan.org, last accessed March 11, 2014). The search yielded 407 miRNAs against aurora kinase A, as predicted by at least two databases. Among these miRNAs, let-7b/d/g, miR23a/b, miR-92a/b, miR-199a-3p, and miR-24 were reported to be differentially expressed in miRNA profiles of $\mathrm{PCa}$ compared with noncancer prostate tissue ${ }^{10,11}$; therefore, we next examined the expression of these miRNAs in human PCa.

\section{miR-199a-3p Expression Is Down-Regulated in Human $\mathrm{PCa}$}

To determine whether the levels of let-7b/d/g, miR-23a/b, miR-92a/b, miR-199a-3p, and miR-24 are down-regulated in clinical PCa, we used quantitative RT-PCR (RT-qPCR) to analyze RNAs from 12 paired benign and tumor human PCa specimens with a Gleason score of $>7$. RNAs were isolated from human tissues, reverse transcribed, and subjected to RT-qPCR. The levels of miR-199a-3p were significantly decreased in 9 of 12 tumors compared with their matched benign prostate tissues (Figure 1A), showing a much greater decrease than the other miRNAs (Table 2). Next, we examined the protein levels of aurora kinase A, the potential target of these miRNAs, via Western blot analysis. We found that aurora kinase A proteins were significantly up-regulated in most of the cancer tissues compared with their matched benign tissues (Figure 1B). Furthermore, the aurora kinase A protein levels were correlated inversely with the miR-199a-3p levels, with a correlation coefficient of 0.938 (Figure 1C). This was much higher than other miRNAs (Table 2), suggesting that aurora kinase A

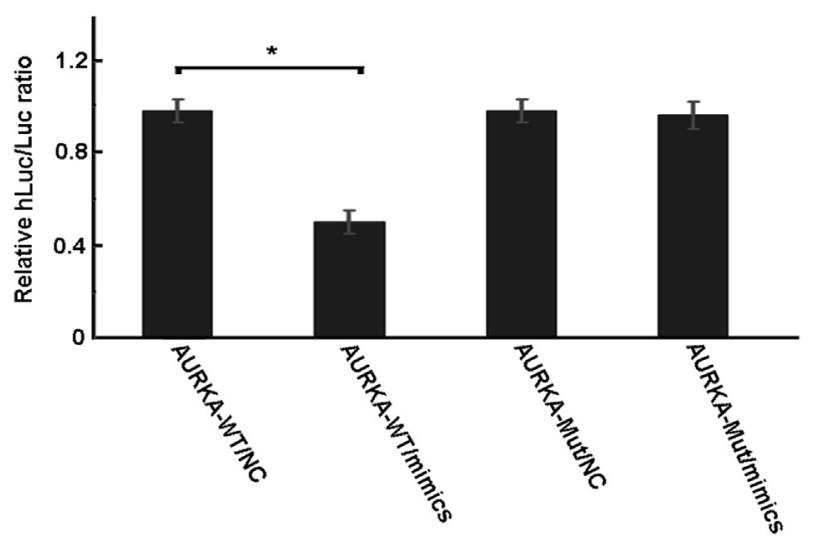

Figure 2 Relative luminescence in plasmid constructs containing miR199a-3p target sites. miR-199a-3p target regions in the $3^{\prime}$-UTR of aurora kinase A were identified using TargetScan (http://www.targetscan.org, last accessed March 11, 2014) and microRNA.org (http://www.microrna.org/ microrna/home.do, last accessed March 11, 2014). The regions were cloned into luciferase reporter plasmids. Mutated $3^{\prime}$-UTR constructs were generated using site-directed mutagenesis. The plasmid construct, together with miR-199a-3p mimics, was cotransfected into HeLa cells. Luminescence readings were obtained 48 hours after transfection. Transfection efficiencies were normalized to hLuc luciferase, which was constitutively expressed by the report vector. Statistical analyses were done using analysis of variance. The data shown are the means $\pm \operatorname{SEM}(N=3)$. ${ }^{*} P<0.05$. AURKA, aurora kinase $A$; NC, noncancerous tissue; WT, wild type.

expression is regulated primarily by miR-199a-3p in human PCa. Thus, in this study, we chose to focus on miR-199a-3p.

\section{Luciferase Reporter Assay}

To validate direct interaction of miR-199a-3p with aurora kinase A mRNAs, their $3^{\prime}$-UTR target site was cloned into luciferase reporter plasmid. This construct was cotransfected with miR-199a-3p mimics into HeLa cells. Cells transfected with miR-199a-3p mimics caused a reduction in luciferase expression (Figure 2). Site-directed mutagenesis of the miR199a-3p recognition site, located on the aurora kinase A

Table 2 Expression of Aurora Kinase A and miRNAs

\begin{tabular}{|c|c|c|c|c|c|c|c|c|c|c|c|c|c|c|}
\hline \multirow[b]{2}{*}{ Gene } & \multicolumn{12}{|c|}{ Relative expression of each case } & \multirow[b]{2}{*}{$R^{2}$} & \multirow[b]{2}{*}{$\mathrm{RD}$} \\
\hline & 1 & 2 & 3 & 4 & 5 & 6 & 7 & 8 & 9 & 10 & 11 & 12 & & \\
\hline AuroraA & 2.2 & 1.8 & 1.0 & 1.9 & 1.9 & 2.4 & 1.1 & 1.6 & 1.9 & 1.0 & 2.1 & 1.9 & - & - \\
\hline let-7d & 0.7 & 1.0 & 0.6 & 1.1 & 0.5 & 0.6 & 0.7 & 1.1 & 0.6 & 0.6 & 1.2 & 0.5 & 0.026 & $8 / 12$ \\
\hline let-7g & 0.7 & 1.1 & 0.7 & 1.1 & 0.6 & 0.5 & 0.7 & 1.1 & 0.5 & 0.7 & 1.1 & 0.6 & 0.000 & $8 / 12$ \\
\hline $\operatorname{miR}-23 a$ & 1.1 & 0.8 & 1.2 & 0.7 & 0.7 & 0.9 & 1.3 & 0.8 & 1.2 & 1.1 & 0.9 & 1.0 & 0.243 & $7 / 12$ \\
\hline $\operatorname{miR}-92 b$ & 0.7 & 1.2 & 1.1 & 0.8 & 0.7 & 0.9 & 1.2 & 1.2 & 1.1 & 0.8 & 0.9 & 0.8 & 0.162 & $7 / 12$ \\
\hline $\operatorname{miR}-199 a-3 p$ & 0.3 & 0.5 & 1.0 & 0.5 & 0.4 & 0.2 & 1.2 & 0.7 & 0.4 & 1.0 & 0.3 & 0.4 & 0.938 & $9 / 12$ \\
\hline $\operatorname{miR}-24$ & 0.6 & 0.8 & 1.1 & 0.9 & 1.3 & 0.3 & 0.7 & 0.7 & 0.5 & 1.1 & 0.5 & 0.4 & 0.333 & $9 / 12$ \\
\hline
\end{tabular}

Relative expression of aurora kinase A protein and miRNAs was detected in the paired benign and malignant prostate tissues. The data of aurora kinase $\mathrm{A}$ protein and miRNA expression in benign or tumor prostate tissues from three independent experiments were averaged, the data for benign tissues were arbitrarily designated as one, and the relative expression was obtained when data for tumor tissues were divided by data for benign tissues.

$\mathrm{RD}$, rate of decrease; -, not applicable. 
A
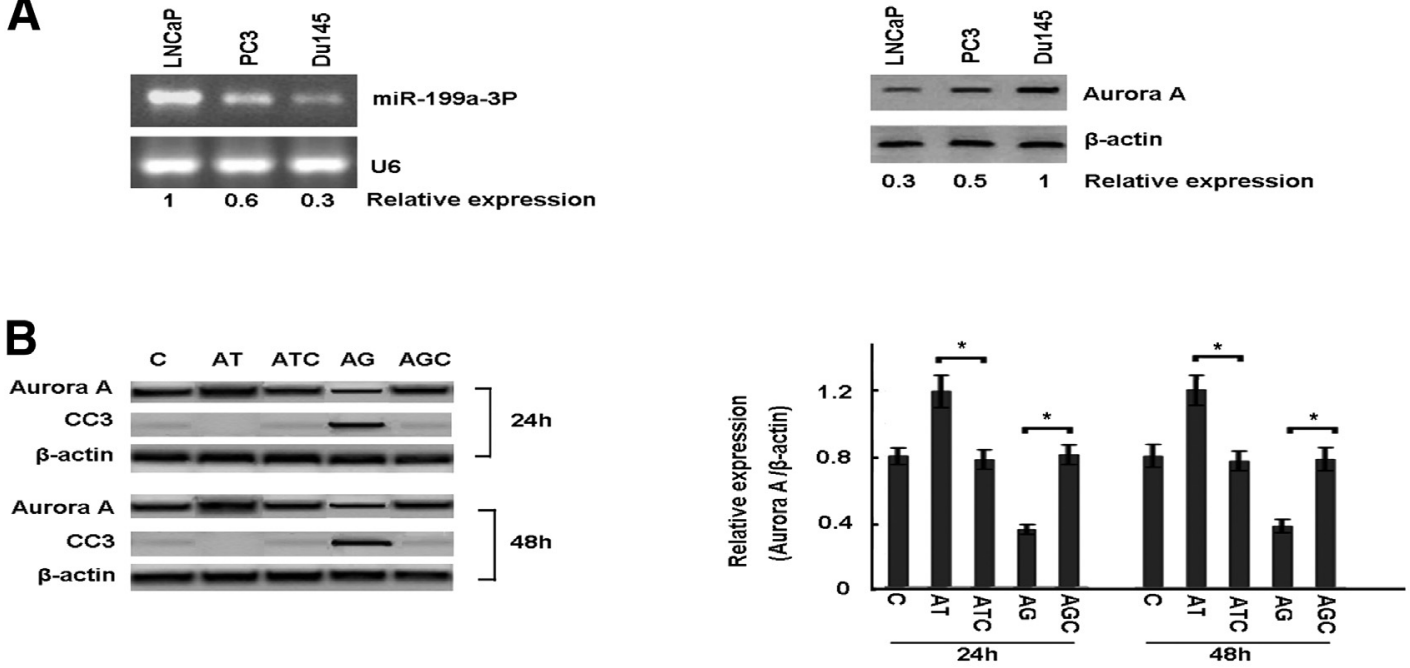

C
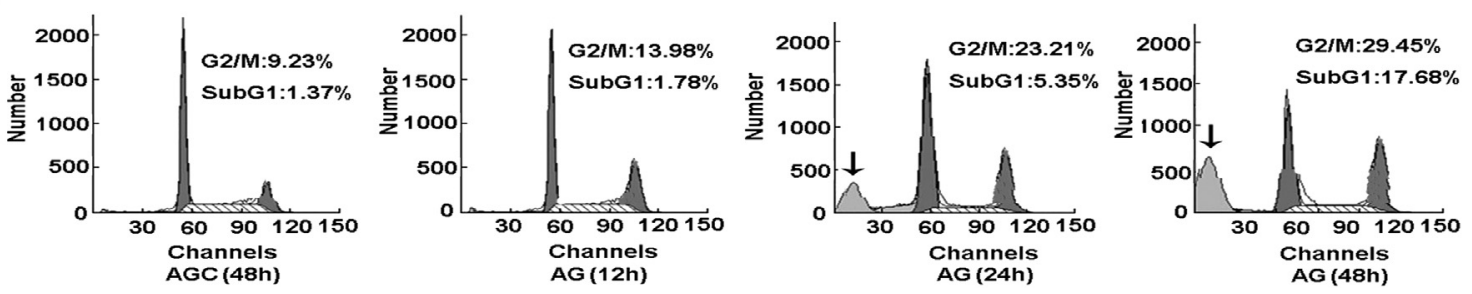

Figure 3 miR-199a-3p agomir induces cell cycle arrest and cell apoptosis. A: The expression of miR-199a-3p and aurora kinase A in PCa cell lines, PC-3, DU145, and LNCaP, detected by RT-PCR and Western blot analysis, shows that the expression of miR-199a-3p is the lowest and that of aurora kinase A is the highest in DU145 cells (normalized to U6 and $\beta$-actin, respectively). miR-199a-3p agomir or antagomir was transfected into DU145 cells, and the expression of aurora kinase A and CC3 was detected by Western blot analysis. Western blot analysis shows that miR-199a-3p agomir significantly decreases aurora kinase A protein, whereas miR199a-3p antagomir significantly increases aurora kinase A protein. B: CC3 was nearly undetectable in control groups, whereas miR-199a-3p agomir obviously increased CC3 protein. C: Inhibition of aurora kinase A arrests cell cycle transition and induces apoptosis. In control, the percentage of cells in $\mathrm{G}_{2}-\mathrm{M}$ was approximately $9.23 \%$ at each time point. When treated with agomir, the percentage of cells in $\mathrm{G}_{2}-\mathrm{M}$ was increased to $13.98 \%$ at $12 \mathrm{hours,} 23.21 \%$ at $24 \mathrm{hours}$, and $29.45 \%$ at 48 hours after incubation. In addition, a fraction of cells in the sub- $\mathrm{G}_{1}$ phase of the cell cycle in the agomir-treated cells was indicated after 48 hours. Arrows indicate cells in the sub- $\mathrm{G}_{1}$ phase. ${ }^{*} P<0.05$. AG, agomir; AGC, agomir control; AT, antagomir; ATC, antagomir control; $C$, vehicle control.

$3^{\prime}$-UTR, abolished interactions between the miRNA and its aurora kinase A targets. This result indicates that miR-199a-3p can directly target aurora kinase A.

\section{miR-199a-3p Agomir Induces Cell Cycle Arrest and Cell Apoptosis}

We examined the expression of miR-199a-3p and aurora kinase A in PCa cell lines PC-3, DU145, and LNCaP, and found that aurora kinase A protein was the highest and miR199a-3p was the lowest in DU145 cells (Figure 3A). To study the regulation of aurora kinase A expression by miR-199a-3p, we transfected the agomir or antagomir of miR-199a-3p into DU145 cells. We found that miR-199a-3p agomir significantly decreased aurora kinase A protein, whereas miR-199a-3p antagomir significantly increased aurora kinase A protein. Cleaved caspase 3 (CC3) was nearly undetectable in control groups, whereas miR-199a-3p agomir obviously increased CC3 protein (Figure 3B). We then examined the effect of miR-199a-3p agomir on cell cycle distribution of DU145 through flow cytometry. In agomir-treated cells, the percentage of cells in $\mathrm{G}_{2}-\mathrm{M}$ was increased to approximately $13.98 \%$ at 12 hours, $23.21 \%$ at 24 hours, and $29.45 \%$ at 48 hours. However, in agomir control-treated cells, the percentage of cells in $\mathrm{G}_{2}-\mathrm{M}$ was approximately $9.23 \%$ at each time point. In addition, we found that a fraction of cells in the sub- $\mathrm{G}_{1}$ phase of the cell cycle (indicating apoptotic cells) in the agomir-treated cells was significantly increased compared with the controltreated cells (Figure 3C).

\section{miR-199a-3p Agomir Inhibits Tumor Growth in Vivo}

To further detect the function of miR-199a-3p agomir in vivo, we set up a tumor model by s.c. transplanting DU145 cells into nude mice. After tumors reached approximately $65 \mathrm{~mm}^{3}$, $10 \mathrm{nmol} / \mathrm{L}$ agomir and agomir control in $30 \mu \mathrm{L}$ PBS or $30 \mu \mathrm{L}$ PBS alone was injected into the tumor tissue every 2 days by multipoint intratumoral injection, lasting for 15 days. We found that agomir significantly inhibited the growth of human $\mathrm{PCa}$ cells as solid tumors in athymic nude mice, whereas control had no effect on tumor growth (Figure 4, A and B). 
A

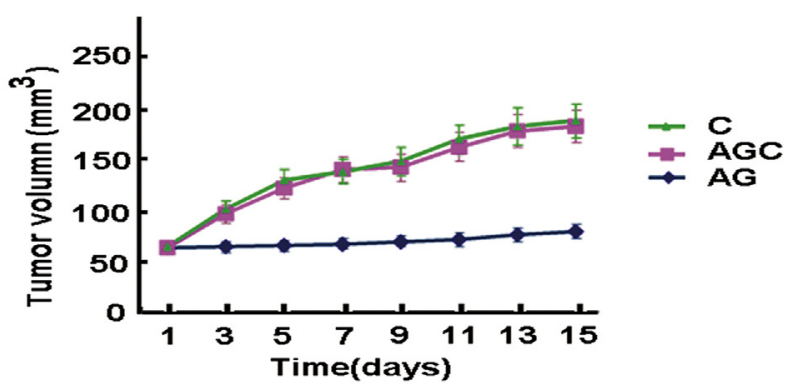

B
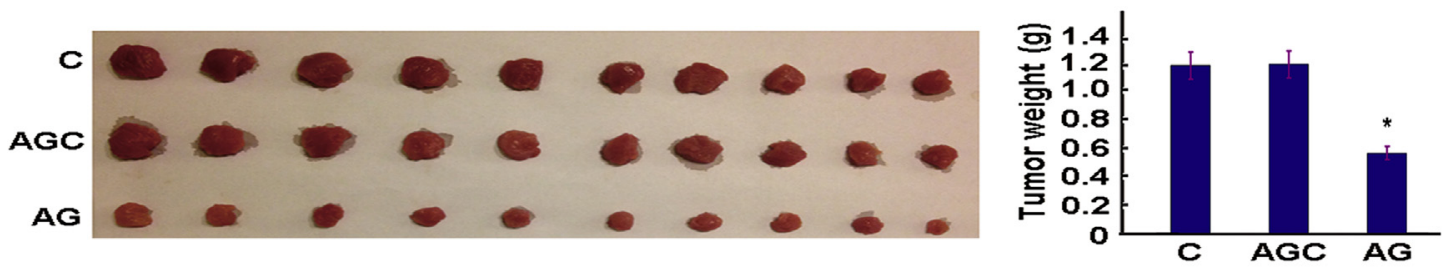

C

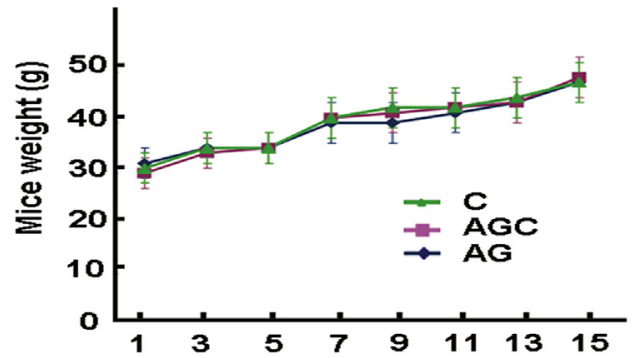

D

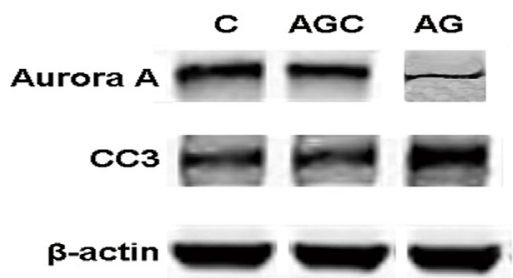

Figure 4 PCa growth in athymic nude mice is inhibited by miR-199a-3p agomir. An in vivo tumor model was set by s.c. transplanting DU145 cells into nude mice. After tumors reached approximately $65 \mathrm{~mm}^{3}$, agomir and agomir control ( $4 \mathrm{nmol} / \mathrm{L}$, respectively) in $30 \mu \mathrm{L}$ PBS or $30 \mu \mathrm{L}$ PBS alone was injected into the tumor tissue every 2 days by multipoint intratumoral injection over the course of 15 days. A: Tumor volume assessment by digital caliper every 2 days shows that the growth of tumors in the agomir group is much slower than in the control groups. B: Mean tumor weight immediately after resection 15 days after commencement of therapy shows that tumor weight in the agomir group is less than that in the control groups. C: Body weight assessment and tumor size evaluation of mice every 2 days shows that agomir treatment does not affect the body weight of mice. D: After 15 days, mice in each group were decapitated and tumor tissues were collected. Western blot analysis was performed to determine the expressions of aurora kinase A and cleaved caspase 3. Agomir was found to significantly inhibit aurora kinase A expression. Conversely, the CC3 in tumor tissues was increased. ${ }^{*}<0.05$. AG, agomir; AGC, agomir control; C, PBS control.

Throughout the study, animals did not exhibit signs of discomfort as the result of tumor growth, and the body weights of the mice were not adversely affected by agomir administration (Figure 4C). We also found that agomir obviously inhibited aurora kinase A expression in tumor tissues (Figure 4D). Conversely, active caspase-3 in tumor tissues was increased (Figure 4D).

\section{AZA Increases Expression of miR-199a-3p in DU145 Cells}

After treatment of DU145 cells with the demethyltransferase inhibitor, AZA, methylation was decreased in the miR-199a gene (Figure 5A), and miR-199a-3p expression was upregulated as determined by RT-PCR (Figure 5B). Aurora kinase A was down-regulated, as detected by Western blot analysis (Figure 5C), in these same DU145 cells, suggesting expression of miR-199a-3p in DU145 cells is methylation dependent.

\section{miR-199a-3p Expression and miR-199a Gene Methylation Correlate With the Malignancy of $\mathrm{PCa}$}

To analyze the relevance of promoter methylation on miR199a-3p expression in PCa, MSP was performed for the 12 cases previously described. The average methylation percentage was $30 \%$ in normal surrounding tissue and $83 \%$ in paired cancer tissue (Figure 6A). This difference leads us to envision a relationship between miR-199a-3p expression, its promoter methylation, and clinical parameters. We then enlarged the sample size to examine this potential relationship. The FFPE prostatic tissues were obtained from The People's Hospital of Sichuan Province. All patients had undergone surgery at the same hospital between 2007 and 2011. A total of $50 \mathrm{PCa}$ samples and 20 normal prostate tissues were acquired. RNA or DNA was extracted from FFPE tissues. miRNA was detected, and bisulfite conversion and MSP reactions were performed as previously described. Comparison of the noncancerous versus malignant groups indicated miR-199a-3p was significantly down- 


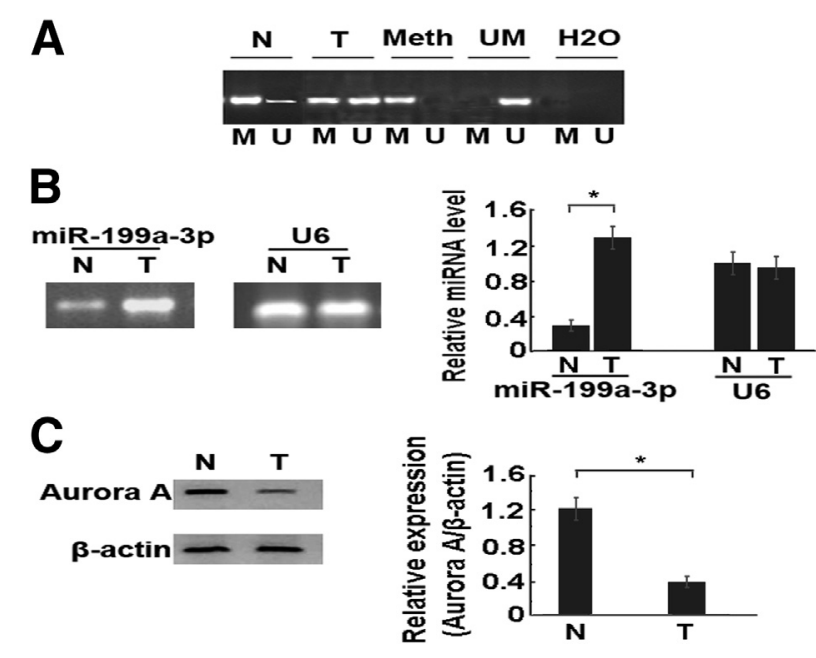

Figure 5 miR-199a-3p expression responses to AZA treatment in DU145 cells. DU145 cells were treated with AZA, then genomic DNA was extracted and subjected to bisulfite modification, and the methylation status of miR$199 a$ gene was detected by qMSP. Moreover, RT-PCR was used to detect miR-199a-3p, and Western blot analysis was used to detect aurora kinase A protein in DU145 cells, with or without AZA treatment. A: MSP shows that methylation is decreased in the miR-199a gene in DU145 cells treated with AZA. B: RT-PCR shows that miR-199a-3p expression is up-regulated in DU145 cells treated with AZA. U6 was used for normalization, and data were presented as fold change compared with U6. C: Western blot analysis shows that aurora kinase A protein is down-regulated in DU145 cells treated with AZA. ${ }^{*} P<0.05$. M, methylated; Meth, methylated positive control; N, no treatment with AZA; $T$, treatment with AZA; $U$, unmethylated; UM, unmethylated positive control.

regulated in malignant tumors (Figure 6B). The relative expression of miR-199a-3p and the methylation of the $m i R$ $199 a$ gene were correlated to tumor stage and Gleason score of PCa (Figure 6, B and C).

\section{Discussion}

Herein, we found that miR-199a-3p was down-regulated in PCa tissues compared with normal prostate tissues, and the expression pattern of miR-199a-3p was inversely correlated with the expression pattern of aurora kinase $\mathrm{A}$, an oncogene that contributes to the malignant phenotypes of PCa. Furthermore, miR-199a-3p agomir attenuated PCa growth, which was concurrent with down-regulation of protein expression of aurora kinase A. Moreover, we demonstrated that miR-199a-3p targeted the aurora kinase A $3^{\prime}$-UTR. Thus, the present study indicates that miR-199a-3p plays a critical role in inhibition of $\mathrm{PCa}$ growth by targeting aurora kinase A.

We also focused on the mechanism by which miR-199a$3 p$ is down-regulated in PCa. miR-199a is regulated by its promoter methylation in testicular cancer, ${ }^{9}$ and we hypothesized that this mechanism also played a role in PCa. We then treated DU145 cells with the demethyltransferase inhibitor, AZA, showing that the unmethylated fraction was increased in the miR-199a promoter after AZA treatment, and that miR-199a-3p expression was up-regulated and aurora kinase A expression was down-regulated. These results suggest miR-199a-3p expression in DU145 cells is methylation dependent. Next, when we examined the relevance of promoter methylation with miR-199a-3p expression in $\mathrm{PCa}$ patients, it showed more methylation in $\mathrm{PCa}$ tissues than in normal tissues, suggesting miR-199a promoter methylation might contribute to cancer malignancy. We then measured miR-199a promoter methylation status and miR-199a-3p expression in an enlarged PCa cohort and evaluated the relationship between miR-199a-3p expression, its promoter methylation, and clinical parameters of $\mathrm{PCa}$. We found that the relative expression of miR-199a-3p and the percentage of methylation were correlated to tumor stage and Gleason score of the PCa, suggesting clinical significance for miR-199a-3p expression and miR-199a promoter methylation in $\mathrm{PCa}$.

Aberrant miR-199a-3p expression has been reported in several cancers; it can be either up-regulated or
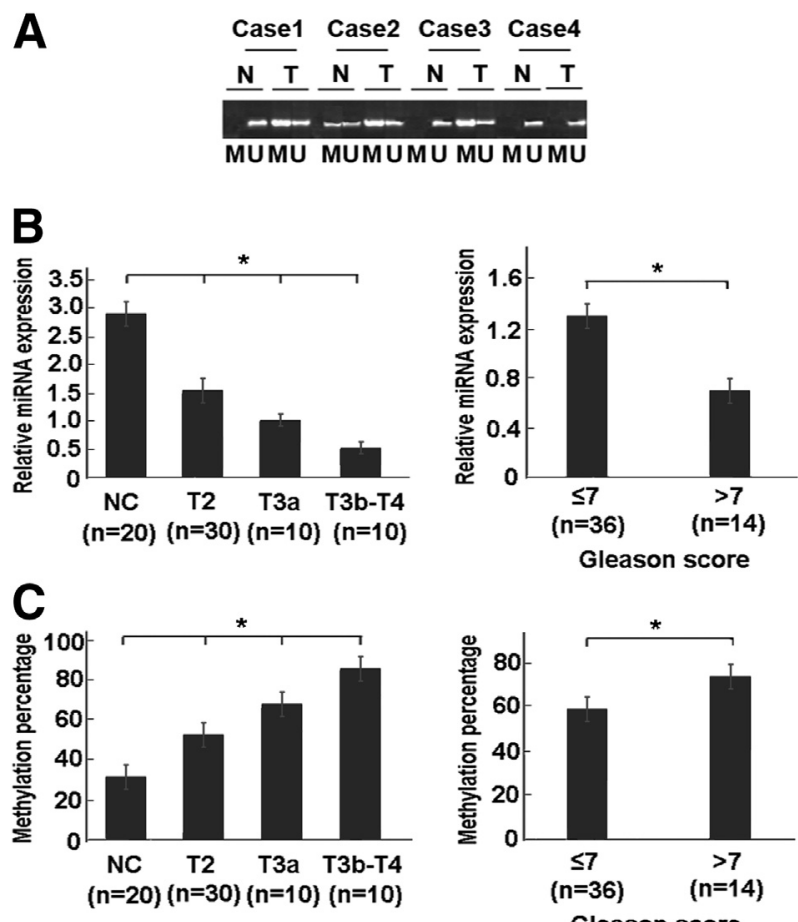

Figure 6 miR-199a-3p expression and miR-199a gene methylation in clinical specimens. Genomic DNA was extracted from normal or tumor prostate tissues and subjected to bisulfite modification, and the methylation status of miR-199a gene was detected by qMSP. RT-PCR was used to detect miR-199a-3p in each sample. A: Representative agarose gel MSP images of four $\mathrm{PCa}$ cases with paired normal surrounding tissues shows that the methylation of miR-199a gene in prostate tumor tissues is much higher than that in normal surrounding tissues. B: Expression of miR-199a-3p in 20 noncancer and $50 \mathrm{PCa}$ tissues shows that miR-199a-3p is decreased with the increasing severity of the malignancy of $\mathrm{PCa}$; the difference between the noncancer and all malignant tissue groups was significant. C: Methylation percentage of miR-199a gene in 20 noncancer and $50 \mathrm{PCa}$ tissues shows that the methylation of miR-199a gene is increased with the increasing severity of the malignancy of $\mathrm{PCa}$; the difference between the noncancer and all malignant tissue groups was significant. ${ }^{*} P<0.05$. $M$, methylated primers; N, normal; NC, noncancerous tissue; T, tumor; T2, T3a, $\mathrm{T} 3 \mathrm{~b}, \mathrm{~T} 4$, malignant stages of $\mathrm{PCa}$; $\mathrm{U}$, unmethylated primers. 
down-regulated in distinct cancer cell types. ${ }^{12}$ Duan et $\mathrm{al}^{13}$ have reported that miR-199a-3p was down-regulated in human osteosarcoma, and that restoring miR-199a-3p's function may provide therapeutic benefits in osteosarcoma. However, Wan et $\mathrm{al}^{14}$ reported that miR-199a-3p expression was significantly up-regulated in human colorectal cancer. The different expression patterns of miR-199a-3p found in different cancers suggests a complicated role for miR-199a$3 p$ in regulating cancer phenotype. Herein, we show, for the first time to our knowledge, that miR-199a-3p is a potent inhibitor of aurora kinase A and it inhibits PCa growth through targeting aurora kinase A. However, we still realize that, although aurora kinase $\mathrm{A}$ may be the primary gene regulated by miR-199a-3p in $\mathrm{PCa}$, we cannot exclude the possibility that other targeted genes of miR-199a-3p might also exert some functions in PCa growth.

Up until now, many inhibitors against aurora kinase A have been developed, such as VX-680, ${ }^{15}$ MLN8054, ${ }^{16}$ and aurora kinase A RNA interference. ${ }^{17}$ However, to our knowledge, this is the first report to demonstrate that miRNA-mediated knockdown of aurora kinase A can be used as a specific gene-targeting therapy to suppress progression of PCa. As we know, miRNAs are endogenously produced molecules in the body and have higher stability and better tissue compatibility compared with other agents ${ }^{7}$; thus, they might be better treatments than currently available therapeutics. Throughout the study, we found that animals did not exhibit signs of discomfort, and the body weights of the mice were not adversely affected by ribonucleotide administration, proving the safety of miRNA administration in mice. Further work should be performed to enhance the delivery ratio of miRNAs/solid tumors to improve their therapeutic effects.

In summary, our findings show that miR-199a-3p is a potent inhibitor of aurora kinase A in PCa. miR-199a-3p agomir effectively attenuates malignant progression of $\mathrm{PCa}$ both in vitro and in vivo. Revealing novel mechanisms for oncogene inhibition by miRNA-mediated pathways offers new avenues for PCa treatment.

\section{Acknowledgment}

We thank Stephanie Cambier for proofreading the manuscript.

\section{References}

1. Bar-Shira A, Pinthus JH, Rozovsky U, Goldstein M, Sellers WR, Yaron Y, Eshhar Z, Orr-Urtreger A: Multiple genes in human 20q13 chromosomal region are involved in an advanced prostate cancer xenograft. Cancer Res 2002, 62:6803-6807

2. Lee EC, Frolov A, Li R, Ayala G, Greenberg NM: Targeting Aurora kinases for the treatment of prostate cancer. Cancer Res 2006, 66: 4996-5002

3. Qu Y, Zhang L, Mao M, Zhao F, Huang X, Yang C, Xiong Y, Mu D: Effects of DNAzymes targeting Aurora kinase A on the growth of human prostate cancer. Cancer Gene Ther 2008, 15:517-525

4. Battel DP: MicroRNAs: genomics, biogenesis, mechanism, and function. Cell 2004, 116:281-297

5. Chua JH, Armugam A, Jeyaseelan K: MicroRNAs: biogenesis, function and applications. Curr Opin Mol Ther 2009, 11:189-199

6. Farazi TA, Hoell JI, Morozov P, Tuschl T: MicroRNAs in human cancer. Adv Exp Med Biol 2013, 774:1-20

7. Sethupathy P, Collins FS: MicroRNA target site polymorphisms and human disease. Trends Genet 2008, 24:489-497

8. Livak KJ, Schmittgen TD: Analysis of relative gene expression data using real-time quantitative PCR and the 2(-Delta Delta C(T)) Method. Methods 2001, 25:402-408

9. Cheung HH, Davis AJ, Lee TL, Pang AL, Nagrani S, Rennert OM, Chan WY: Methylation of an intronic region regulates miR-199a in testicular tumor malignancy. Oncogene 2011, 30:3404-3415

10. Porkka KP, Pfeiffer MJ, Waltering KK, Vessella RL, Tammela TL, Visakorpi T: MicroRNA expression profiling in prostate cancer. Cancer Res 2007, 67:6130-6135

11. Szczyrba J, Löprich E, Wach S, Jung V, Unteregger G, Barth S, Grobholz R, Wieland W, Stöhr R, Hartmann A, Wullich B, Grässer F: The microRNA profile of prostate carcinoma obtained by deep sequencing. Mol Cancer Res 2010, 8:529-538

12. Sakurai K, Furukawa C, Haraguchi T, Inada K, Shiogama K, Tagawa T, Fujita S, Ueno Y, Ogata A, Ito M, Tsutsumi Y, Iba H: MicroRNAs miR-199a-5p and -3p target the Brm subunit of SWI/SNF to generate a double-negative feedback loop in a variety of human cancers. Cancer Res 2011, 71:1680-1689

13. Duan Z, Choy E, Harmon D, Liu X, Susa M, Mankin H, Hornicek F: MicroRNA-199a-3p is downregulated in human osteosarcoma and regulates cell proliferation and migration. Mol Cancer Ther 2011, 10: $1337-1345$

14. Wan D, He S, Xie B, Xu G, Gu W, Shen C, Hu Y, Wang X, Zhi Q, Wang L: Aberrant expression of miR-199a-3p and its clinical significance in colorectal cancers. Med Oncol 2013, 30:378

15. Harrington EA, Bebbington D, Moore J, Rasmussen RK, Ajose-Adeogun AO, Nakayama T, Graham JA, Demur C, Hercend T, Diu-Hercend A, Su M, Golec JM, Miller KM: VX-680, a potent and selective small-molecule inhibitor of the Aurora kinases, suppresses tumor growth in vivo. Nat Med 2004, 10:262-267

16. Manfredi MG, Ecsedy JA, Meetze KA, Balani SK, Burenkova O, Chen W, Galvin KM, Hoar KM, Huck JJ, LeRoy PJ, Ray ET, Sells TB, Stringer B, Stroud SG, Vos TJ, Weatherhead GS, Wysong DR, Zhang M, Bolen JB, Claiborne CF: Antitumor activity of MLN8054, an orally active small-molecule inhibitor of Aurora A kinase. Proc Natl Acad Sci U S A 2007, 104:4106-4111

17. Hata T, Furukawa T, Sunamura M, Egawa S, Motoi F, Ohmura N, Marumoto T, Saya H, Horii A: RNA interference targeting aurora kinase a suppresses tumor growth and enhances the taxane chemosensitivity in human pancreatic cancer cells. Cancer Res 2005, 65: 2899-2905 